\title{
Patient-specific instruments as a standard procedure in total knee arthroplasty: Logistics and postoperative radiological results in 70 patients
}

\author{
Dominik Drnek ${ }^{*}$ Nicolas Haffner, Armin Sadjed, Peter Ritschl \\ Vienna Hospital Association, Orthopedic Clinic Gersthof, Vienna, Austria; *Corresponding Author: dominik.drnek@wienkav.at
}

Received 30 October 2013; revised 27 November 2013; accepted 20 December 2013

Copyright (c) 2014 Dominik Drnek et al. This is an open access article distributed under the Creative Commons Attribution License, which permits unrestricted use, distribution, and reproduction in any medium, provided the original work is properly cited. In accordance of the Creative Commons Attribution License all Copyrights (C 2014 are reserved for SCIRP and the owner of the intellectual property Dominik Drnek et al. All Copyright (C) 2014 are guarded by law and by SCIRP as a guardian.

\section{ABSTRACT}

Serous Purpose: There is limited information in the literature surrounding the use of patientspecific instrumentation (PSI) by a large number of surgeons. This prospective observational study was therefore designed to evaluate the logistics of using PSI for total knee arthroplasty (TKA) in a multi-surgeon environment, as well as its accuracy in positioning components. Methods: Of 73 patients enrolled in this study, 3 were excluded after the surgeon decided intraoperatively to switch to conventional instrumentation. Results: Mean operative time was 77.6 minutes. The component size had to be changed in 19 patients and bone cuts corrected in 12 . In 65 of the 70 cases (92.9\%), mechanical alignment was within the optimal range from $3^{\circ}$ varus to $3^{\circ}$ valgus, with the remaining 5 cases considered outliers (7.1\%). Mean overall mechanical alignment was $0.3^{\circ}$ varus (standard deviation, $\pm 2^{\circ}$ ). There was a distinct variance with regard to the position of the tibial component in the sagittal plane. Conclusions: PSI can be effectively incorporated in larger, multisurgeon practices. Although high accuracy was observed for overall mechanical alignment and component positioning in the frontal and sagittal planes, further attention must be paid to the tibial slope. We highly recommend the use of the extramedullary alignment rod to the proper position of the tibial block, as well as doublechecking the slope before performing bone cuts.

\section{KEYWORDS}

Patient-Matched Instrumentation;

\section{Patient-Matched Cutting Blocks; Total Knee} Arthroplasty; Radiological Results

\section{INTRODUCTION}

Total knee arthroplasty (TKA) is the treatment of choice in moderate-to-severe osteoarthritis of the knee. Although TKA has been a tremendous therapeutic success, complications such as aseptic loosening, instability, dislocation, infection or periprosthetic fracture still occur in approximately $5 \%-8 \%$ of patients [1-3]. Aseptic loosening in particular remains a common cause of revision in TKA [4-6]. Given that malpositioning of the components is a major contributor to aseptic loosening, correct alignment is of paramount importance for the longevity of the prosthesis, as has been shown by Ritter et al. [7].

Patient-specific instrumentation (PSI) was recently developed to address this concern. The process for creating PSI begins with obtaining the patients' preoperative imaging data either through CT or MRI (sometimes supplemented with a long leg standing $\mathrm{X}$-ray), which is then used to create 3-dimensional (3D) reconstructions of the joint surface. Thereafter, 3D drill guides are generated defining the position of conventional cutting blocks or patient-specific cutting blocks for the distal femoral as well as for the tibial cut.

In order to avoid malpositioning of these blocks or drill guides, they are manufactured to have a glove-like fit to the joint surface. Hence, there should only be a possible way of fitting them on the distal femur or the proximal tibia, respectively. In terms of frontal, axial, and rotational alignment, they are oriented to either the mechanical or the anatomical axis [8]. PSI can therefore be considered as navigational TKA, in that it offers preoperative image-based, bone-referenced navigation.

One of the advantages of PSI is the reduction of fur- 
ther surgical steps to determine the 3D alignment of the implant. Furthermore, the preoperative planning includes the correct component size and positioning. Due to the fact that the intramedullary canal is not violated, a number of potential complications, such as fat embolisms and enhanced intraoperative bleeding, are reduced [9].

The logistics of the preoperative planning for PSI differs greatly depending on whether it is performed by a single surgeon or a group of surgeons, the latter of which presumably reflects the reality of most orthopedic departments. A strong relationship with the patient is likely to exist when the initial indication, preoperative planning, and the operation itself are overseen and performed by a single surgeon. However, this is simply not the case if an entire orthopedic department decides to implement PSI as a standard for routine TKA.

We therefore sought to report on our experiences with the implementation of PSI at our institution. This prospective study assesses the logistics we had to deal with, and observes the postoperative radiological results we obtained when using PSI.

\section{PATIENTS AND METHODS}

At the Orthopedic Clinic Gersthof, a PSI system utilizing patient-matched cutting blocks ([PMCB]; VISIONAIRE $^{\circledR}$, Smith \& Nephew $^{\circledR}$ ) was applied. The preoperative image modality was based on a long leg standing X-ray as well as an MRI of the knee. The Genesis II ${ }^{\circledR}$ endoprosthesis (Smith \& Nephew ${ }^{\circledR}$ ) was used as the TKA implant. All patients signed an agreement for data transfer prior to participation in the study, and agreed to the time required for preoperative planning and manufacture of the PMCB.

In contrast to a single surgeon setup, implementing PSI as a standard operating procedure for a larger group of orthopedic surgeons requires additional critical steps. Firstly, we established limits on the indication so that only cruciate-retaining (CR) and posterior-stabilized (PS) TKAs were enrolled in the PSI planning process. The deformity of the arthritic knee was required to be within $15^{\circ}$ of valgus and $15^{\circ}$ of varus and the laxity of the knee joint was also taken into account. This was of paramount importance because the indication for TKA was done at the outpatient clinic by a surgeon who might not have been among the final operating surgeons. Secondly, the following preferences for femur and tibial resection were defined at our institutional account, in accordance with the manufacturer's planning protocol. For the distal femur resection, the perpendicular cut to the mechanical axis was chosen. The trans-epicondylar axis (TEA) landmark was prioritized for rotational alignment of the femur. The other two common landmarks (posterior condylar axis and AP axis [Whiteside line]) were additionally used for rotational alignment. The distal resec- tion height was determined by the implant thickness of $9.5 \mathrm{~mm}$. For the anterior and posterior femoral cuts, we used an anterior referenced system with an optional APshift for the best flexion/extension gap. In cases where the calculated size was in between two sizes, the component was to be downsized in order to avoid an overhang of the implant, potentially causing soft tissue irritation. This preference was defined for both components (femur and tibia). The anterior cut was too be as flush as possible with the flange level to the anterior femoral cortex, yet slight notching was accepted in order to obtain a well-balanced TKA. The proximal resection of the tibia was planned according to the standard inlay height of $9 \mathrm{~mm}$. The slope was calculated according to the patient's anatomy, with respect to the $4^{\circ}$ of slope already built into the polyethylene (PE). For example, if the patient's slope was measured to be $10^{\circ}$ preoperatively, the cut was performed with a slope of $6^{\circ}$. Rotational alignment of the tibial base plate was fixed to the medial third of the tibial tubercle. After the imaging data was transferred to the manufacturer, the preoperative plan was generated by an engineer and sent to our clinic two weeks later. A further critical step was the short time period (two working days) for obtaining approval of the surgical plan so that PMCB production could begin. We used a single account at our institution, which was administrated by the department head's secretary. Checking and, if necessary, modifying the proposed surgical plan was done by the surgeon on duty. Generally, they were unaware of the patient's clinical situation, but knew of the agreed limits of the indication. As soon as the planning was approved, a report was sent to the manufacturer and within two additional weeks the PMCBs were produced and delivered. Just prior to the operation, a surgeon (who might not have been involved in the planning procedure) checked the patient's clinical situation and the planning data. If they were not satisfied, it was possible to overrule the process and switch to the conventional technique.

The following parameters were registered during surgery: the fit of the cutting blocks, changes of the implant size, bony recuts, soft tissue releases, changes in inlay size and operation time. Gap balancing was subjectively judged by the operating surgeon. The number of blood transfusions and early postoperative complications during the patient's stay at hospital were recorded as well. If no complications occurred, the patients were discharged from hospital 12 days after operation, which is a common procedure at our institution in order to provide an extensive program of physical therapy. The postoperative $\mathrm{X}$-rays (AP, lateral view, and long leg standing) taken 7-to-10 days after surgery were evaluated in terms of component position and restoration of the mechanical alignment. X-rays were routinely monitor-guided but not 
calibrated at our institution.

From September 2011 to October 2012, 73 patients were enrolled in our study and underwent TKA. Three patients were excluded from our analysis because the surgeon decided intraoperatively to switch to the conventional instrumentation. In one patient, the PMCB did not fit due to a mix up of patient's imaging data by the manufacturer. In the two other patients the surgeon found that the femoral block was not appropriate in terms of rotational alignment.

We maintained loose inclusion criteria for this trial and did not exclude patients for reasons such as previous operations, varus or valgus deformities, extension or flexion contracture, high body mass index, or other illnesses like diabetes that might have influenced the outcome. Patients with contraindications for the preoperative MRI (e.g., those with pacemakers) were excluded from the study. Hence, the study group of 70 patients consisted of 48 women and 22 men between 47 and 90 years of age (mean 70, standard deviation [SD] \pm 9.7 ). Their body mass index ranged from 21.2 to 41.8 (mean, 29.2, SD \pm 4.6 ). The underlying cause for TKA was non-inflammatory osteoarthritis in 68 cases, rheumatoid arthritis in one case and avascular necrosis of the medial femoral condyle (Ahlbeck's disease) in the other case. Twenty-three patients had flexion contractures ranging from $5^{\circ}$ to $20^{\circ}$ preoperatively. The preoperative deformity measured by the Hip-Knee-Ankle Angle was between $12^{\circ}$ varus and $15^{\circ}$ valgus, with a mean of $3.4^{\circ}$ varus (SD $\left.\pm 5.8^{\circ}\right)$. Nine patients had previous operations on the ipsilateral hip (seven THA and two osteosynthetic devices).

The surgical approach consisted of a standard medial parapatellar arthrotomy. The rest of the operation followed the principles of a bone-referenced femur-first technique. According to the protocol, the osteophytes were not resected to gain the best fit for the cutting block. When attaching the PMCBs to the joint surface, care was taken to drill through the pin holes in the proper axis to avoid altering the cutting block's position. Holes were drilled through the joint surface first and consequently two conventional pins were applied. Thereafter, two drill pins for the anterior pinholes were used to secure the block's position. After the bone cuts had been performed spacer blocks were used to allow perfect gap sizing. If needed, soft tissue releases were carried out in order to balance the knee.

Statistical analyses: The calculation of means and standard deviations was performed using Microsoft ${ }^{\circledR}$ Excel 2010 for Windows ${ }^{\circledR}$.

\section{RESULTS}

Seventy patients underwent TKA using this PSI technology. A CR design was chosen in 68 cases and a PS design in the remaining 2 . The mean operative time was 76.6 minutes (range, 47 - 113, SD \pm 15.3).

The following changes to the suggested planning with regards to the inlay height, bony cutting level, and implant size were observed. In $25.7 \%$ of cases the standard PE (9 mm) had to be changed. In the CR subgroup, insert was increased to $11 \mathrm{~mm}$ in 14 patients due to insufficient ligamentous stability. Two additional patients in that subgroup received a $13 \mathrm{~mm}$ and a $15 \mathrm{~mm}$ PE insert, respectively. In the PS subgroup, an $11 \mathrm{~mm}$ and an $18 \mathrm{~mm}$ PE was implanted. The latter was necessary as the cutting level of the tibia had to be distalized due to a large osseous defect of the medial condyle.

In one patient, the level of the distal femoral cut was proximalized by $2 \mathrm{~mm}$ due to the extension gap being too narrow. In 10 cases, the tibial cut was distalized 2 $\mathrm{mm}$ in order to address the tightness of the flexion and extension gap. Soft tissue releases were performed in 10 patients. On the medial side, the medial collateral ligament (MCL) was released four times and in two cases a combined release of the MCL and the posteromedial corner (PMC) was performed. In one case all medial structures had to be released (MCL, PMC and the semimembranosus muscle). Laterally, the iliotibial band was released two times, and the posterolateral corner was released in one patient.

Changes of the size of at least one component were carried out in 19 patients. The size of the femoral component was altered four times and the size of the tibial implant 18 times.

Changes to the suggested planning performed during the operation are shown in Table 1.

The average mechanical axis was $0.3^{\circ}\left(\mathrm{SD} \pm 2^{\circ}\right)$ varus. A postoperative alignment within $\pm 3^{\circ}$ varus/valgus was achieved in 65 out of 70 patients (92.9\%). In the remaining five patients (7.1\%), outlier mechanical axes of $3.5^{\circ}$, $4^{\circ}, 4^{\circ}$, and $6^{\circ}$ varus, and $5^{\circ}$ valgus were measured (Table 2).

The positions of the femoral and the tibial component were evaluated separately. The femoral component was implanted at $0.2^{\circ}$ varus ( $\mathrm{SD} \pm 1.5^{\circ}$ ) on average. Optimal results $0^{\circ} \pm 2^{\circ}$ varus/valgus were seen in 62 of 70 cases (88.6\%). In the sagittal plane, the femoral component position was in $4.2^{\circ}$ flexion on average ( $\mathrm{SD} \pm 2.2^{\circ}$ ), ranging from $0^{\circ}$ to $9^{\circ}$. An optimal sagittal alignment of $4^{\circ}$ of flexion $\pm 2^{\circ}$ of the femoral component was found in 53 of 70 patients $(75.7 \%)$. Overstuffing, measured at the very upper end of the femoral shield, was seen in 13 cases (mean $2 \mathrm{~mm}$, maximum $4.5 \mathrm{~mm}$ ) and caused by an increased flexion of the femoral component.

Notching occurred in 5 cases (mean $2.2 \mathrm{~mm}$ ) with a maximum of $3.5 \mathrm{~mm}$. In 2 of these cases, femoral component size was downsized. The average tibial component alignment in the frontal plane was $0.1^{\circ}$ valgus (SD \pm 
Table 1. Changes required during surgery $(n=70)$.

\begin{tabular}{|c|c|c|c|}
\hline Femur & Change made & Number & Percentage \\
\hline \multirow[t]{5}{*}{ Size } & upsized +2 & 0 & 0 \\
\hline & upsized +1 & 0 & 0 \\
\hline & no changes & 66 & $94.3 \%$ \\
\hline & downsized -1 & 4 & $5.7 \%$ \\
\hline & downsized -2 & 0 & 0 \\
\hline \multirow[t]{3}{*}{ Cutting level } & $2 \mathrm{~mm}$ up & 1 & $1.5 \%$ \\
\hline & no changes & 69 & $98.5 \%$ \\
\hline & $2 \mathrm{~mm}$ down & 0 & 0 \\
\hline Tibia & Change made & Number & Percentage \\
\hline \multirow[t]{5}{*}{ Size } & upsized +2 & 2 & $2.9 \%$ \\
\hline & upsized +1 & 7 & $10 \%$ \\
\hline & no changes & 52 & $74.3 \%$ \\
\hline & downsized -1 & 8 & $11.4 \%$ \\
\hline & downsized -2 & 1 & $1.4 \%$ \\
\hline \multirow[t]{3}{*}{ Cutting level } & $2 \mathrm{~mm}$ up & 0 & $0 \%$ \\
\hline & no changes & 60 & $85.7 \%$ \\
\hline & $2 \mathrm{~mm}$ down & 10 & $14.3 \%$ \\
\hline Insert & Change made & Number & Percentage \\
\hline \multirow[t]{6}{*}{ Height } & no change $9 \mathrm{~mm}$ CR & 52 & $74.4 \%$ \\
\hline & $11 \mathrm{~mm} \mathrm{CR}$ & 14 & $20 \%$ \\
\hline & $11 \mathrm{~mm}$ PS & 1 & $1.4 \%$ \\
\hline & $13 \mathrm{~mm} \mathrm{CR}$ & 1 & $1.4 \%$ \\
\hline & $15 \mathrm{~mm} \mathrm{CR}$ & 1 & $1.4 \%$ \\
\hline & $18 \mathrm{~mm}$ PS & 1 & $1.4 \%$ \\
\hline Release & Soft tissue & Number & Percentage \\
\hline none & & 60 & $85.7 \%$ \\
\hline \multirow[t]{3}{*}{ medial } & medial collateral ligament & 4 & $5.7 \%$ \\
\hline & mcl + posteromedial corner & 2 & $2.9 \%$ \\
\hline & $\mathrm{mcl}+\mathrm{pmc}+\mathrm{M}$. semimembranosus & 1 & $1.4 \%$ \\
\hline \multirow[t]{2}{*}{ lateral } & iliotibial band & 2 & $2.9 \%$ \\
\hline & posterolateral corner & 1 & $1.4 \%$ \\
\hline
\end{tabular}


Table 2. Frontal plane alignment.

\begin{tabular}{cccc}
\hline & Mean $( \pm$ Standard deviation) & Number of outliers $^{* *}$ & Percentage of outliers $^{*}$ \\
\hline HKA $^{*}$ angle & $0.3^{\circ}$ varus $\left( \pm 2^{\circ}\right)$ & 5 & $7.1 \%$ \\
\hline Femur & Mean $( \pm$ Standard deviation) & Number of outliers $^{* * * *}$ & Percentage of outliers $^{\circ}$ \\
Tibia & $0.2^{\circ}$ varus $\left( \pm 1.5^{\circ}\right)$ & 8 & $11.4 \%$ \\
\hline
\end{tabular}

${ }^{*}$ Hip Knee Ankle, ${ }^{* *}>3^{\circ}$ varus/valgus, ${ }^{* * *}>2^{\circ}$ varus/valgus.

$1.8^{\circ}$ ). Optimal results $0^{\circ} \pm 2^{\circ}$ varus/valgus were seen in 54 of 70 cases $(77.1 \%)$. In the sagittal plane, we observed some malpositioning of the tibial tray. The average gain of slope compared with the patient's anatomy prior to surgery was $1.7^{\circ}\left(\mathrm{SD} \pm 3.8^{\circ}\right)$. The mean difference between the patient's preoperative slope and the postoperative result was $3.3^{\circ}$ with a maximum deviation of $11^{\circ}$. As a result, the postoperative slope varied from $18^{\circ}$ posterior to $1^{\circ}$ anterior slope (including the $4^{\circ}$ slope embedded in the PE). In the latter case, the patient suffered from a delay in gaining flexion of the knee. (Figure 1)

Seven patients needed blood transfusions, with 12 units of red blood cells transfused to the patients during their stay at our hospital. Two patients had a wound healing deficit-one of them had to undergo a secondary wound closure in the operation room. One patient suffered from a bilateral pulmonary embolism a few days after surgery, even though anticoagulation treatment was provided. No further complications were observed in our sample prior to discharge from the hospital.

\section{DISCUSSION}

The VISIONAIRE® technology used in this study is a preoperative image-based, bone-referenced navigation tool, which ultimately generates a 3D model (PMCB) for the distal femoral and tibial cut. These blocks determine all bony resections, as well as the alignment in all planes (sagittal, rotational, and frontal). This and other PSI technologies are postulated to help surgeons place the prosthesis in a correct position and restore the mechanical axis. According to the literature, this is a key factor for the longevity of the implant $[10,11]$.

Our experience using this PSI technology was somewhat unique, in that we are a department with a large number of surgeons. To our knowledge, the implementation of this technology in a centre such as ours has not yet been detailed in the literature. This unmet need directly led to the first goal of this prospective observational study, which was simply to share the logistics of how we implemented this technology in order that it may be of use to surgeons in similar circumstances.

In a preliminary radiological evaluation of our first 10 cases one anterior slope as well as one severely reduced
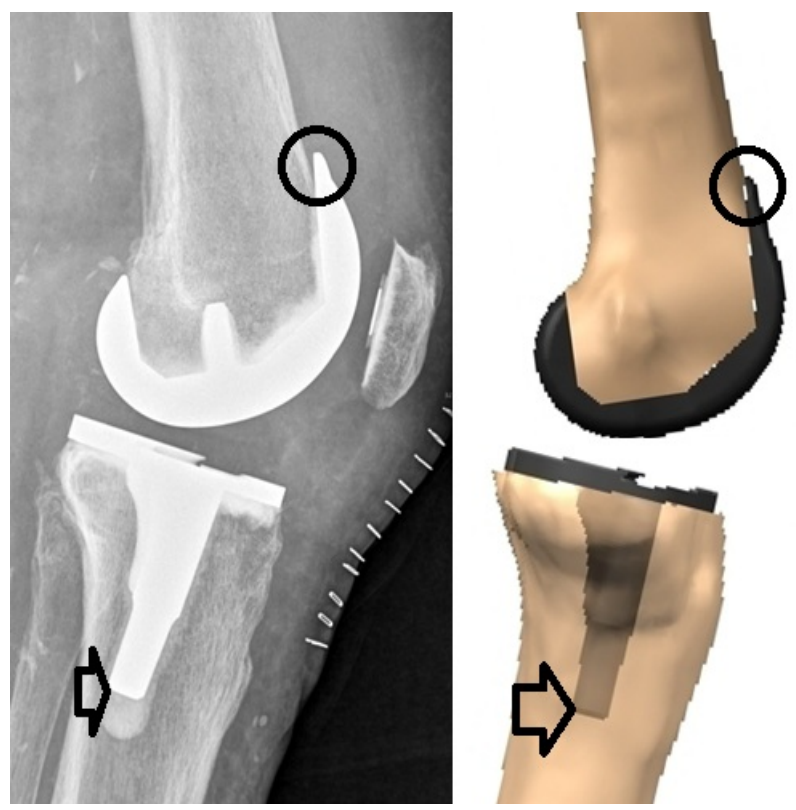

Figure 1. The preoperative planning image shows a normal posterior tibial slope and a femoral component flush with the anterior cortex of the femur. Intraoperatively, the correct position of the PMCB could not be achieved and ultimately resulted in an anterior slope. The alignment rod supplied to avoid malpositioning was not used in this case. In addition, an overstuffing of $2 \mathrm{~mm}$ of the femoral component occurred as shown below. In this case, the preoperative planning differed from the postoperative results.

slope was observed. Possible reasons for that could be that the contact areas of the tibial cutting block on the tibial joint surface are much smaller compared to the ones on the femur. This increases the risk of sliding and tilting of the block on the joint surface and may therefore lead to malpositioning. Secondly, remaining soft tissue at the anterior contact area might interfere with a proper fit of the cutting block. Hence, we recommend accurately removing soft tissues at the anterior border of the tibia before fitting the PMCB.

Drilling through the pin holes in an oblique axis might also alter the cutting block's position. Another possible reason for changes of the tibial slope is that PMCBs, which are made out of nylon, are much more flexible than the conventional metal ones. Hence, the slope can deviate a few degrees if non-directional forces are ap- 
plied to the saw blade during the cutting process, even if all pins are installed properly.

With those experiences in mind, we started using the manufacturer-supplied alignment rod on the tibial cutting block to avoid malpositioning. In our opinion the rod is of great help for double-checking the slope, as well as the block's position. We generally observed that the smaller the contact area of the blocks, the easier it was for malalignment of the block to occur.

The second aim of this study was to gauge whether PSI technology, in combination with this logistical and preoperative planning strategy, would result in positive radiological outcomes when measured postoperatively. We were encouraged to observe only a $7.1 \%$ rate of outliers in the frontal plane in our series. This was somewhat surprising, given that this result was achieved in a multi-surgeon setting.

A recent study evaluating the frontal plane alignment using the same VISIONAIRE ${ }^{\circledR}$ technology found outliers in 11 of 100 (11\%) knees [12]. Similar results were reported by Ng et al. in a single-surgeon trial of 105 TKAs using a different PSI system (Signature ${ }^{\circledR}$, Biomet $^{\circledR}$ ) compared with 55 TKAs using ordinary instrumentation. The number of outliers was $8.6 \%$ in the PSI group compared with $21.8 \%$ in the conventional instrumentation group [13]. These numbers are also comparable to those found by Mason et al. in his meta-analysis of computer-assisted TKA [14].

On the contrary, Barrack et al. compared 100 TKAs with standard instrumentation to 100 TKAs with customcutting guides (Signature ${ }^{\circledR}$, Biomet ${ }^{\circledR}$ ), and found that there was no statistically significant difference in the number of outliers in the coronal plane between the two consecutively enrolled groups (31\% in the PSI group vs. $23 \%$ in the conventional group) [15].

The reported disadvantages of PSI are that the blocks do not fit accurately, and that the proposed size of the components and the pre-planned resection height does not match the intraoperative findings. Stronach recorded 161 intraoperative changes in 66 knee arthroplasties using the Signature ${ }^{\circledR}$ PSI, equalling 2.4 changes per knee. Implant sizes were changed in $77 \%$ of the femurs and $53 \%$ of the tibias in this trial. The guide did not fit securely on $12 \%$ of the femurs and $5 \%$ of the tibias. Conventional instrumentation had to be used in $5 \%$ of the femurs and $8 \%$ of the tibias due to the poor fit or obviously inaccurate proposed resections [16].

In our series of 70 knees using the VISIONAIRE ${ }^{\circledR}$ technique, we observed $5.7 \%$ of the femurs and $25.7 \%$ of the tibias changed in size. In $4.1 \%$ of the patients (3 of the 73 patients enrolled), conventional instrumentation had to be used.

There are important limitations to our study worth mentioning. Firstly, the study was not comparative in nature. Our goal was more simple, namely to relate our department's experience implementing PSI as a standardized and routine operation in a large group of orthopedic surgeons, and to compare our radiological results to the current available literature. Secondly, we only evaluated PSI from one manufacturer in spite of the fact that multiple manufacturers offer this technology. Separate manufacturer's block designs, as well as their imaging modalities and protocols, may differ widely. In the literature today, there is only one study comparing a conventional technique with two separate MRI-based PSI systems: the Signature system by Biomet ${ }^{\circledR}$, which aims to restore the mechanical axis, and the OtisMed ${ }^{\circledR}$ system, which aligns implants according to the kinematical axis. The study found no reduction in outliers concerning the coronal alignment: $16 \%$ in the conventional group vs. $18 \%$ in the Signature ${ }^{\circledR}$ group and $44 \%$ in the OtisMed ${ }^{\circledR}$ group [17]. Further comparative studies are required to gauge the effect of different image modalities and block designs on radiological outcomes.

\section{CONCLUSION}

In our multi-surgeon setup, TKAs performed using PSI achieved results comparable to those observed in the literature with navigated techniques with regard to the number of outliers in frontal alignment. Due to some inaccuracies of the tibial tray in the sagittal plane, we strongly recommend the use of the supplied alignment rod to position the tibial cutting block properly as well as to check the slope before performing the cut.

\section{ETHICS}

All procedures followed were in accordance with the ethical standards of the responsible committee on human experimentation (institutional and national) and with the Helsinki Declaration of 1975, as revised in 2008.

\section{INFORMED CONSENT}

Informed consent was obtained from all patients for being included in the study.

\section{CONFLICT OF INTEREST}

Dominik Drnek, Nicolas Haffner, and Armin Sadjed declare that they have no conflict of interest.

Peter Ritschl is a consultant of Smith \& Nephew ${ }^{\circledR}$. No financial support has been received or will be received from a commercial party related directly or indirectly to the subject of this article.

\section{REFERENCES}

[1] Aglietti, P and Buzzi, R. (1988) Posteriorly stabilised 
total-condylar knee replacement. Three to eight years' follow-up of 85 knees. The Bone \& Joint Journal, 70, 211-216.

[2] Stern, S.H. and Insall, J.N. (1992) Posterior stabilized prosthesis. Results after follow-up of nine to twelve years. The Journal of Bone \& Joint Surgery, American Volume, 74, 980-986.

[3] Vince, K.G., Insall, J.N. and Kelly, M.A. (1989) The total condylar prosthesis. 10- to 12-year results of a cemented knee re-placement. The Journal of Bone \& Joint Surgery (British Volume), 71, 793-797.

[4] Fehring, T.K., Odum, S., Griffin, W.L., Mason, J.B. and Nadaud, M. (2001) Early failures in total knee arthroplasty. Clinical Orthopaedics and Related Research, 392, 315-318. http://dx.doi.org/10.1097/00003086-200111000-00041

[5] Ritter, M.A., Faris, P.M., Keating, E.M. and Meding, J.B. (1994) Postoperative alignment of total knee replacement. Its effect on survival. Clinical Orthopaedics and Related Research, 299, 153-156.

[6] Sharkey, P.F., Hozack, W.J., Rothman, R.H., Shastri, S. and Jacoby, S.M. (2002) Insall Award paper. Why are total knee arthroplasties failing today? Clinical Orthopaedics and Related Research, 404, 7-13. http://dx.doi.org/10.1097/00003086-200211000-00003

[7] Ritter, M.A., Davis, K.E., Meding, J.B., Pierson, J.L., Berend, M.E. and Malinzak, R.A. (2011) The effect of alignment and BMI on failure of total knee replacement. The Journal of Bone \& Joint Surgery, American Volume, 93, 1588-1596. http://dx.doi.org/10.2106/JBJS.J.00772

[8] Nam, D., McArthur, B.A., Cross, M.B., Pearle, A.D., Mayman, D.J. and Haas, S.B. (2012) Patient-specific instrumentation in total knee arthroplasty: A review. The Journal of Knee Surgery, 25, 213-219. http://dx.doi.org/10.1055/s-0032-1319785

[9] Church, J.S., Scadden, J.E., Gupta, R.R., Cokis, C., Williams, K.A. and Janes, G.C. (2007) Embolic phenomena during computer-assisted and conventional total knee replacement. The Journal of Bone \& Joint Surgery (British Volume), 89, 481-485. http://dx.doi.org/10.1302/0301-620X.89B4.18470
[10] Fang, D.M., Ritter, M.A. and Davis, K.E. (2009) Coronal alignment in total knee arthroplasty: Just how important is it? The Journal of Arthroplasty, 24, 39-43. http://dx.doi.org/10.1016/j.arth.2009.04.034

[11] Jeffery, R.S., Morris, R.W. and Denham, R.A. (1991) Coronal alignment after total knee replacement. The Journal of Bone \& Joint Surgery (British Volume), 73, 709714.

[12] Daniilidis, K. and Tibesku, C.O. (2013) Frontal plane alignment after total knee arthroplasty using patient-specific instruments. International Orthopaedics, 37, 45-50. http://dx.doi.org/10.1007/s00264-012-1732-1

[13] Ng, V.Y., DeClaire, J.H., Berend, K.R., Gulick, B.C. and Lombardi, Jr., A.V. (2012) Improved accuracy of alignment with patient-specific positioning guides compared with manual instrumentation in TKA. Clinical Orthopaedics and Related Research, 470, 99-107. http://dx.doi.org/10.1007/s11999-011-1996-6

[14] Mason, J.B., Fehring, T.K., Estok, R., Banel, D. and Fahrbach, K. (2007) Meta-analysis of alignment outcomes in computer-assisted total knee arthroplasty surgery. The Journal of Arthroplasty, 22, 1097-1106. http://dx.doi.org/10.1016/j.arth.2007.08.001

[15] Barrack, R.L., Ruh, E.L., Williams, B.M., Ford, A.D., Foreman, K. and Nunley, R.M. (2012) Patient specific cutting blocks are currently of no proven value. The Journal of Bone \& Joint Surgery (British Volume), 94-B, 95-99. http://dx.doi.org/10.1302/0301-620X.94B11.30834

[16] Stronach, B.M., Pelt, C.E., Erickson, J. and Peters, C.L. (2013) Patient-specific total knee arthroplasty required frequent surgeon-directed changes. Clinical Orthopaedics and Related Research, 471, 169-174. http://dx.doi.org/10.1007/s11999-012-2573-3

[17] Nunley, R.M., Ellison, B.S., Zhu, J., Ruh, E.L., Howell, S.M. and Barrack, R.L. (2012) Do patient-specific guides improve coronal alignment in total knee arthroplasty? Clinical Orthopaedics and Related Research, 470, 895902. http://dx.doi.org/10.1007/s11999-011-2222-2 